Portland State University

PDXScholar

Mechanical and Materials Engineering Faculty

Publications and Presentations

Mechanical and Materials Engineering

$4-2009$

\title{
Gravity Effects on Capillary Flows in Sharp Corners
}

Enrique Ramé

Mark M. Weislogel

Portland State University, weisloge@pdx.edu

Follow this and additional works at: https://pdxscholar.library.pdx.edu/mengin_fac

Part of the Fluid Dynamics Commons, and the Materials Science and Engineering Commons

Let us know how access to this document benefits you.

Citation Details

Ramé, E., \& Weislogel, M. M. (2009). Gravity effects on capillary flows in sharp corners. Physics Of Fluids, $21(4), 042106$.

This Article is brought to you for free and open access. It has been accepted for inclusion in Mechanical and Materials Engineering Faculty Publications and Presentations by an authorized administrator of PDXScholar. Please contact us if we can make this document more accessible: pdxscholar@pdx.edu. 


\title{
Gravity effects on capillary flows in sharp corners
}

\author{
Enrique Ramé ${ }^{1}$ and Mark M. Weislogel ${ }^{2}$ \\ ${ }^{1}$ National Center for Space Exploration Research in Fluids and Combustion, \\ clo NASA Glenn Research Center, MS 110-3, Cleveland, Ohio 44135, USA \\ ${ }^{2}$ Department of Mechanical and Materials Engineering, Portland State University, Portland, \\ Oregon 97201, USA
}

(Received 25 September 2008; accepted 26 February 2009; published online 21 April 2009)

\begin{abstract}
We analyze the effect of gravity on capillary flows in sharp corners. We consider gravity perpendicular and parallel to the channel axis. We analyze both steady and unsteady flows. In the steady analysis the main result is a closed form expression for the flow rate as a function of the two gravity components. Good agreement with steady experiments is offered as support of the model. The unsteady analysis is restricted to "small" values of the two gravity parameters and is accomplished using a similarity formulation. The similarity coefficients of the gravity corrections are fully determined by the coefficients of the gravityless problem. The main result of the unsteady analysis is the gravity corrections to the flow rate (or rate of advance) of the liquid in the channel. In addition, we obtain corrections for the liquid height as a function of position and time. We address in detail unsteady problems with select boundary conditions that are representative of typical flow types. In Appendix A we present a new exact solution to one of the gravityless similarity cases, which is analogous to a nonlinear heat conduction equation. In Appendix B we offer dimensional formulas for all the unsteady flow results, which are valuable for systems design and analysis.
\end{abstract}

(C) 2009 American Institute of Physics. [DOI: 10.1063/1.3109685]

\section{INTRODUCTION}

When liquid in a reservoir is placed in contact with a sharp corner of angle $2 \alpha$, a liquid filament is driven from the reservoir into the channel provided that the Concus-Finn condition $^{1}$ is met between the static contact angle $\theta$ and the corner angle, i.e., $\theta<\pi / 2-\alpha$. In Fig. 1 we show a schematic of such a flow. In zero gravity, the flow is in the direction of decreasing filament height $h$. This is due to the capillary pressure, which in systems that meet the Concus-Finn condition, is smaller than the ambient gas pressure and decreases as the liquid height decreases. The presence of gravity may reinforce or oppose the capillary pressure driving force.

The capillary flow of liquids in sharp corners in the absence of gravity has been studied extensively by Ayyaswamy et al., ${ }^{2}$ Ransohoff and Radke, ${ }^{3}$ Dong and Chatzis, ${ }^{4}$ Romero and Yost, ${ }^{5}$ Kovscek and Radke, ${ }^{6}$ Weislogel, ${ }^{7}$ and Weislogel and Lichter ${ }^{8}$ (W\&L in what follows) among many others, both analytically and experimentally. These studies lay the mathematical foundations and offer extensive experimental verification. The analysis is made possible by approximating the total curvature by the cross sectional curvature and, as by W\&L, by a choice of scales that absorbs most of the geometrical effects of varying contact angle and corner angle.

The effect of gravity pointing normal to the channel has been examined by Weislogel ${ }^{7}$ but only for one type of boundary condition upstream. Bico and Quéré ${ }^{9}$ analyzed capillary rise dynamics of liquids in vertical corner channels with gravity aligned with the channel. The foam drainage equation (FDE) contains the effect of gravity pointing along the channel, and has been studied extensively (see, e.g., Verbist et al. ${ }^{10}$ and Cox and Verbist ${ }^{11}$ ). In the conventional treatment of the FDE, the gravity length scale is absorbed in the scaling of the partial differential equation, leaving the gravity parameter (a kind of Bond number) in the boundary condition for the area of liquid at the inlet. The "traveling wave" solutions represent the wetting of an initially dry foam by liquid injected into the inlet at the top at constant flow rate. These solutions approach a constant liquid area asymptotically at the inlet, implying that the flow there is all gravity driven since no capillary pressure gradient can occur when the liquid flow area is independent of position. One of W\&L's solutions, obtained with $a=0$ in their notation, has a constant liquid area at the flow channel origin and approaches a constant area at upstream infinity. However, even this case is not analogous to the FDE. In the FDE, liquid injected at fixed rate at upstream infinity feeds the volume increase rate downstream. In the case analyzed by W\&L, the flow is not gravity driven as in the FDE but purely capillary. Since at upstream infinity the capillary flow is zero, and there is no fresh liquid injection as in the FDE, the filament moves forward not by injecting liquid but by decreasing the volume stored upstream of the origin. Therefore, traveling wave solutions cannot be compared to flows examined by W\&Land by extension, here-where a capillary driving force exists everywhere.

In this work we examine the effects of gravity on such flows. We analyze steady and unsteady flows. In the latter, we restrict the analysis to conditions that make two appropriate dimensionless groups small-representing, respectively, the importance of gravity versus surface tension in directions parallel and perpendicular to the channel. The small-gravity assumption allows one to seek approximate solutions using a regular perturbation in each of the small parameters. When the zero-gravity boundary value problem un- 


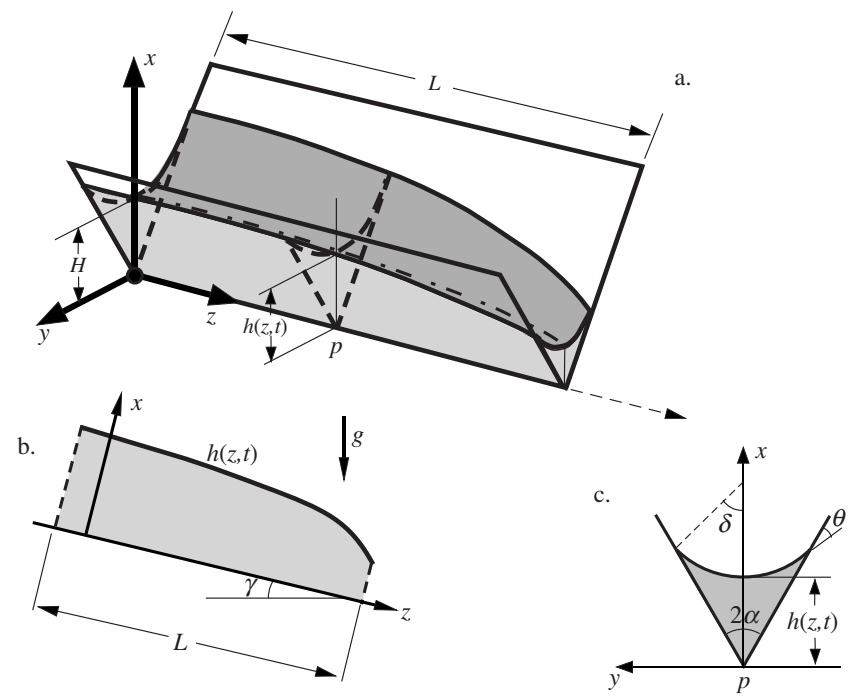

FIG. 1. (a) Schematic of the fluid configuration as it moves down the corner of angle $2 \alpha$, from high to low liquid heights. (b) Schematic of $h(z, t)$ in the $x-z$ plane. The channel vertex forms an angle $\gamma$ with the horizontal. (c) In the $x-y$ plane, the free surface meets the solid with (static) contact angle $\theta$.

steady flow admits similarity solutions, the boundary value problems that describe the gravity corrections (both for gravity perpendicular and parallel to the flow direction) also admit similarity solutions with similarity variables that are fully determined by the boundary condition upstream.

\section{FORMULATION}

We begin by writing the conservation of mass equation (à la "foam equation," ") in dimensionless form for an incompressible liquid of density $\rho$, viscosity $\mu$ and surface tension $\sigma$ flowing in the corner with liquid cross sectional area $A(z, t)$ and volumetric flow rate $Q(z, t)$ (where $z$ is the downstream direction parallel to the corner and $t$ is time). The formulation follows that of $\mathrm{W} \& \mathrm{~L}^{8},{ }^{8}$ and we refer the reader to that paper for additional details. Using scales as by W\&L (see Table I) the dimensionless mass balance may be expressed as volume conservation as

$$
\frac{\partial A}{\partial t}=-\frac{\partial Q}{\partial z}
$$

The flow rate is $Q \equiv\langle w\rangle A$, where the area-averaged axial velocity is

$$
\langle w\rangle \equiv \frac{1}{A} \int_{A(z, t)} w(z, t) d A .
$$

TABLE I. Scaling scheme used by W\&L and in this work.

\begin{tabular}{lcc}
\hline \hline Lengths & Velocities & Other \\
\hline$x^{\prime}, h^{\prime} \rightarrow H$ & $u^{\prime} \rightarrow W H / L$ & $P^{\prime} \rightarrow \sigma / H f$ \\
$z^{\prime} \rightarrow L$ & $v^{\prime} \rightarrow W \tan \alpha H / L$ & $t^{\prime} \rightarrow L / W$ \\
$y^{\prime} \rightarrow H \tan \alpha$ & $w^{\prime} \rightarrow W$ & $A^{\prime} \rightarrow H^{2} \tan \alpha$ \\
$\mathcal{L}^{\prime} \rightarrow L$ & $W=H \sigma \sin ^{2} \alpha / L \mu f$ & $Q^{\prime} \rightarrow W H^{2} \tan \alpha$ \\
\hline \hline
\end{tabular}

Using the same scalings, we write the $z$-component of the linear momentum equation,

$$
0=\nabla_{\mathrm{II}} w-\frac{\partial P}{\partial z}+\frac{f \text { Bo } \sin \gamma}{\epsilon},
$$

where $\quad \nabla_{\mathrm{II}}(.) \equiv \partial(.)^{2} / \partial x^{2}+\partial(.)^{2} / \partial y^{2}, \quad P \quad$ is pressure, $f \equiv \sin \alpha /[\sin (\alpha+\delta)-\sin \alpha] \quad$ (with $\delta \equiv \pi / 2-\alpha-\theta$ ), $\mathrm{Bo} \equiv \rho g H^{2} / \sigma$, the angle $\gamma$ is measured clockwise from the horizontal to the solid (refer to Fig. 1), and $\epsilon \equiv H / L$ (ratio of vertical to axial scales). This analysis is valid in the limit $\epsilon^{2} \ll 1$ with $f$ being held fixed, which allows us to neglect inertia and gradients in the $z$-direction. In this limit, the condition $\epsilon^{2} f \ll 1$ is also obeyed, which makes the axial interface curvature negligible relative to the curvature in the $x-y$ plane.

From Eq. (3), we may write using dimensional analysis

$$
\langle w\rangle=h^{2} F_{i}\left(-\frac{\partial P}{\partial z}+G_{z}\right),
$$

where $F_{i}$ is only a function of the cross section geometry and is identical to the one calculated by $\mathrm{W} \& \mathrm{~L},{ }^{8}$ and $G_{z}$ $\equiv f$ Bo $\sin \gamma / \epsilon$.

We now proceed to generate an equation for $h(z, t)$. We do this in two steps. First, we write the pressure (see Ref. 8) as $P=-1 / h+f$ Bo $\cos \gamma(h-x)$, hence

$$
P_{z}=\left(\frac{1}{h^{2}}+G_{x}\right) \frac{\partial h}{\partial z},
$$

where $G_{x} \equiv f$ Bo cos $\gamma$. Second, we replace Eq. (5) into Eq. (4), which, when combined with $Q=\langle w\rangle A$ and using $A \sim h^{2}$, may be replaced into Eq. (1) to get

$$
\frac{\partial h^{2}}{\partial t}+\frac{\partial}{\partial z}\left\{F_{i} h^{4}\left[-\left(\frac{1}{h^{2}}+G_{x}\right) \frac{\partial h}{\partial z}+G_{z}\right]\right\} .
$$

Clearly, flow with gravity can develop even when the Concus-Finn condition is not obeyed.

For nonzero gravity, the cross sectional shape-and, thus, $F_{i}$-depends not only on $\alpha$ and $\delta$, but also on $G_{x}$, the dimensionless component of gravity normal to the channel axis. Anticipating the fact that we will pursue an asymptotic analysis valid as $G_{x} \rightarrow 0$, we note that it is possible, in principle, to generate by computation the coefficients of the asymptotic expansion

$$
F_{i}\left(\alpha, \delta, G_{x}\right) \sim F_{i}(\alpha, \delta, 0)+\left.G_{x} \frac{\partial F_{i}}{\partial G_{x}}\right|_{(\alpha, \delta, 0)}+\cdots,
$$

where $\left(\partial F_{i} / \partial G_{x}\right)(\alpha, \delta, 0)$ is calculable numerically by performing the operation

$$
\left.\frac{\partial F_{i}}{\partial G_{x}}\right|_{(\alpha, \delta, 0)} \approx \frac{F_{i}\left(\alpha, \delta, G_{x}\right)-F_{i}(\alpha, \delta, 0)}{G_{x}}
$$

and taking the limit of the result as $G_{x} \rightarrow 0$. Nevertheless, as will be discussed subsequently, we expect the dependence of $F_{i}$ on $G_{x}$ to be weak. Thus, in the present analysis we will neglect this dependence and take $F_{i}\left(\alpha, \delta, G_{x}\right)$ as a given datum. In this context, the analysis will focus on the gravity effect on the hydrostatic pressure and on the capillary pressure driving force-not on the weak effect of gravity on the 


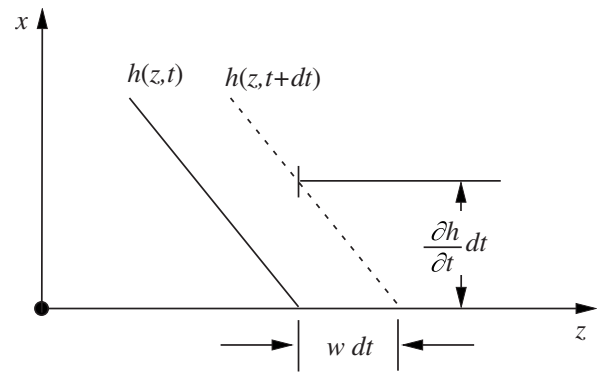

FIG. 2. Schematic of the tip region of the liquid advancing in the channel. The lines at an angle to the axes represent the limiting slope of the interface positions at $t$ and at $t+d t$. The liquid occupies the space to the left of the line at each time. The intersections with the $z$-axis correspond to $\mathcal{L}(t)$ and $\mathcal{L}(t$ $+d t)$.

flow resistance $F_{i}$. Effecting the time derivative in Eq. (6), and treating $F_{i}$ as independent of $G_{x}$, we may define $\tau$ $\equiv t F_{i} / 2$ to get

$$
\begin{aligned}
\frac{\partial h}{\partial \tau}= & 2\left(\frac{\partial h}{\partial z}\right)^{2}+h \frac{\partial^{2} h}{\partial z^{2}}+G_{x}\left[4\left(h \frac{\partial h}{\partial z}\right)^{2}+h^{3} \frac{\partial^{2} h}{\partial z^{2}}\right] \\
& -4 G_{z} h^{2} \frac{\partial h}{\partial z} .
\end{aligned}
$$

The boundary condition at the tip is zero height,

$$
h\left(z, \tau ; G_{x}, G_{z}\right)=0 \quad \text { at } \quad z=\mathcal{L}\left(\tau ; G_{x}, G_{z}\right),
$$

where $z=\mathcal{L}\left(\tau ; G_{x}, G_{z}\right)$ is the location of the moving liquid tip and is part of the solution. Determining how $\mathcal{L}\left(\tau ; G_{x}, G_{z}\right)$ depends on $G_{x}$ and $G_{z}$ is a main goal of this work.

At $z=0$ we may impose several different height or volumetric flow rate boundary conditions. Since the problem conserves volume, the volumetric inflow at $z=0$ must be equal to the volume change in the fluid in the channel

$$
\left.\int_{0}^{t} Q\right|_{z=0} d t^{\prime}=\left.\frac{2}{F_{i}} \int_{0}^{\tau} Q\right|_{z=0} d \tau^{\prime}=\int_{0}^{\mathcal{L}} A d z .
$$

When $\left.Q\right|_{z=0}$ is given as a datum, Eq. (11) provides an additional condition to be satisfied. Alternatively, the height at $z=0$ may be prescribed as a function of time, demanding that

$$
h(0, \tau)=\mathcal{H}(\tau),
$$

where $\mathcal{H}(\tau)$ is given.

\section{A. Properties of Eq. (9)}

Two properties are important in this analysis. First is the relation at the tip $z=\mathcal{L}(\tau)$ (where the dependence on $G_{x}$ and $G_{z}$ is not written out but should be understood) between the slope $\partial h / \partial z$ and $\partial h / \partial \tau$. Evaluating Eq. (9) at the tip by setting $h=0$, we get

$$
\left.\frac{\partial h}{\partial \tau}\right|_{z=\mathcal{L}(\tau)}=\left.2\left(\frac{\partial h}{\partial z}\right)^{2}\right|_{z=\mathcal{L}(\tau)} .
$$

Any solution to Eq. (9) must satisfy Eq. (13). This condition may also be derived geometrically from a kinematic condition that holds at the tip of any interface moving along a solid. In Fig. 2 we can see that from kinematic consider- ations,

$$
\frac{\partial h}{\partial t} d t=-\frac{\partial h}{\partial z} w d t, \quad \text { at } z=\mathcal{L}(t) .
$$

Since at $z=\mathcal{L}(t), w=\langle w\rangle=-h_{z} F_{i}$, and recalling that $\tau=t F_{i} / 2$, condition (13) follows.

The second property derives from the volume conservation Eq. (11). If we integrate Eq. (6) from 0 to $\mathcal{L}$, we get

$$
\frac{1}{2} \frac{\partial}{\partial \tau} \int_{0}^{\mathcal{L}} h^{2} d z=-\left.h^{2} \frac{\partial h}{\partial z}\right|_{0}-\left.G_{x} h^{4} \frac{\partial h}{\partial z}\right|_{0}-\left.G_{z} h^{4}\right|_{0} .
$$

If we integrate between 0 and $\tau$, we get

$$
\int_{0}^{\mathcal{L}} h^{2} d z=-\left.2 \int_{0}^{\tau}\left(h^{2} \frac{\partial h}{\partial z}+G_{x} h^{4} \frac{\partial h}{\partial z}+G_{z} h^{4}\right)\right|_{z=0} d \tau^{\prime} .
$$

This condition, derived from the mass conservation equation, states that the instantaneous liquid volume at time $\tau$ (the left-hand side) equals the volume influx through the plane $z=0$. It may be also derived from Eq. (11) by expressing $Q$ in terms of the field variables [see Eq. (4)].

\section{STEADY FLOWS}

\section{A. Steady theory}

Since the channel is defined for $0 \leq z \leq 1$, and demand that $h(0)=H_{0}$ and $h(1)=H_{1}$, the steady solution to Eq. (6) can be obtained implicitly in closed form

$$
\begin{aligned}
z G_{z}= & G_{x}\left(h-H_{0}\right)+\frac{1}{2 C^{1 / 4}}\left[( 1 - \sqrt { C } G _ { x } ) \left(\arctan \frac{h}{C^{1 / 4}}\right.\right. \\
& \left.-\arctan \frac{H_{0}}{C^{1 / 4}}\right)-\left(1+\sqrt{C} G_{x}\right)\left(\operatorname{artanh} \frac{h}{C^{1 / 4}}\right. \\
& \left.\left.-\operatorname{artanh} \frac{H_{0}}{C^{1 / 4}}\right)\right]
\end{aligned}
$$

where the value of the constant $C$ can be found implicitly from

$$
\begin{aligned}
G_{z}= & G_{x}\left(H_{1}-H_{0}\right)+\frac{1}{2 C^{1 / 4}}\left[( 1 - \sqrt { C } G _ { x } ) \left(\arctan \frac{H_{1}}{C^{1 / 4}}\right.\right. \\
& \left.-\arctan \frac{H_{0}}{C^{1 / 4}}\right)-\left(1+\sqrt{C} G_{x}\right)\left(\operatorname{artanh} \frac{H_{1}}{C^{1 / 4}}\right. \\
& \left.\left.-\operatorname{artanh} \frac{H_{0}}{C^{1 / 4}}\right)\right] .
\end{aligned}
$$

This solution is valid for $C>h^{4}$, implying that $G_{z}^{-1}(d h / d z)$ $<0$ for $0<z<1$. On the other hand, when $0<C<h^{4}$ in 0 $<z<1$, the first derivative meets the constraint $G_{z}^{-1}(d h / d z)$ $>0$ and the solution is 


$$
z G_{z}=G_{x}\left(h-H_{0}\right)+\frac{1}{2 C^{1 / 4}}\left[\left(1-\sqrt{C} G_{x}\right)\left(\arctan \frac{h}{C^{1 / 4}}-\arctan \frac{H_{0}}{C^{1 / 4}}\right)-\left(1+\sqrt{C} G_{x}\right)\left(\operatorname{arcoth} \frac{h}{C^{1 / 4}}-\operatorname{arcoth} \frac{H_{0}}{C^{1 / 4}}\right)\right],
$$

and $C$ is obtained from

$$
G_{z}=G_{x}\left(H_{1}-H_{0}\right)+\frac{1}{2 C^{1 / 4}}\left[\left(1-\sqrt{C} G_{x}\right)\left(\arctan \frac{H_{1}}{C^{1 / 4}}-\arctan \frac{H_{0}}{C^{1 / 4}}\right)-\left(1+\sqrt{C} G_{x}\right)\left(\operatorname{arcoth} \frac{H_{1}}{C^{1 / 4}}-\operatorname{arcoth} \frac{H_{0}}{C^{1 / 4}}\right)\right] .
$$

When $C<0, C$ is determined by

$$
\begin{aligned}
G_{z}= & G_{x}\left(H_{1}-H_{0}\right)+\frac{\sqrt{2}}{4(-C)^{1 / 4}}\left\{\left(1-\sqrt{-C} G_{x}\right)\left[\arctan \frac{\sqrt{2}(-C)^{1 / 4} H_{1}}{\sqrt{-C}-H_{1}^{2}}-\arctan \frac{\sqrt{2}(-C)^{1 / 4} H_{0}}{\sqrt{-C}-H_{0}^{2}}+K \pi\right]\right. \\
& \left.-\left(1+\sqrt{-C} G_{x}\right)\left[\operatorname{artanh} \frac{\sqrt{2}(-C)^{1 / 4} H_{1}}{\sqrt{-C}+H_{1}^{2}}-\operatorname{artanh} \frac{\sqrt{2}(-C)^{1 / 4} H_{0}}{\sqrt{-C}+H_{0}^{2}}\right]\right\},
\end{aligned}
$$

and $z=Z(h)$ is obtained by replacing $z$ for $L$ and $h$ for $H_{1}$. Here,

$$
K= \begin{cases}0, & -C<H_{0}^{4} \text { and }-C<H_{1}^{4}, \text { or }-C>H_{0}^{4} \text { and }-C>H_{1}^{4}, \\ 1, & H_{0}^{4}<-C<H_{1}^{4}, \\ -1, & H_{1}^{4}<-C<H_{0}^{4} .\end{cases}
$$

Equation (6) might suggest that $d h / d z=0$ at some $0<z^{*}$ $<1$ such that $h^{4}=C$ at $z=z^{*}$. However, this possibility must be ruled out because each solution branch assumes that $C$ is either larger or smaller than $h^{4}$ everywhere in $0<z<1$; and once the sign of $d h / d z$ has been set, it cannot change. These solutions contain the steady solution presented in Ref. 10 for given flow rate upstream at $z \rightarrow-\infty$, which can be recovered by setting $H_{0}=0$ and letting $0 \leq h \leq C^{1 / 4}$ in Eq. (17) or $C^{1 / 4}$ $\leq h<\infty$ in Eq. (19). In our problem, the domain has finite length and this demands $h^{4} \neq C$. Knowing $C$ from Eq. (18), (20), and (21), the steady flow rate is given by

$$
Q=\frac{F_{i} F_{A} G_{z} C}{\tan \alpha},
$$

where $F_{A} \equiv f^{2}[\cos \theta \sin \delta / \sin \alpha-\delta]$ is a geometrical coefficient between dimensional area and height, $A^{\prime}=F_{A} h^{\prime 2}$.

It is easy to see that when $G_{z} \rightarrow 0, C \rightarrow \infty$ if $Q$ is to remain finite. If we define $\hat{C} \equiv C G_{z}$ so that $Q=F_{i} F_{A} \hat{C} / \tan \alpha$, we have

$$
1 \sim \frac{5\left(H_{0}^{3}-H_{1}^{3}\right)+3 G_{x}\left(H_{0}^{5}-H_{1}^{5}\right)}{15 \hat{C}} \text { as } \quad G_{z} \rightarrow 0 .
$$

This equation determines $\hat{C}$ for known $H_{0}$ and $H_{1}$. If we replace the left-hand side with $z$ and $H_{1}$ with $h$, the equation determines $z(h)$,

$$
z \sim \frac{5\left(H_{0}^{3}-h^{3}\right)+3 G_{x}\left(H_{0}^{5}-h^{5}\right)}{5\left(H_{0}^{3}-H_{1}^{3}\right)+3 G_{x}\left(H_{0}^{5}-H_{1}^{5}\right)} \quad \text { as } \quad G_{z} \rightarrow 0 .
$$

Eliminating $C$ between Eqs. (23) and (24), we get the flow rate in terms of the inlet and outlet liquid heights, as that by Weislogel ${ }^{12}$ (where one may find several other useful closed form solutions for steady and unsteady corner flows),

$$
Q \sim \frac{F_{i} F_{A}}{\tan \alpha} \frac{5\left(H_{0}^{3}-H_{1}^{3}\right)+3 G_{x}\left(H_{0}^{5}-H_{1}^{5}\right)}{15} \text { as } \quad G_{z} \rightarrow 0 .
$$

Examination of Eq. (18) shows that in the limit $H_{1}$ $\rightarrow H_{0}, C$ must take on a certain form. To reveal this form, let

$$
\begin{aligned}
& C(\varepsilon) \sim C_{0}+\varepsilon C_{1}+\cdots, \\
& h(z, \varepsilon) \sim h_{0}(z)+\varepsilon h_{1}(z)+\cdots, \\
& \varepsilon \equiv H_{0}-H_{1}, \quad \varepsilon \rightarrow 0 .
\end{aligned}
$$

Substituting into Eq. (18), we find

$$
\begin{aligned}
& C_{0}=H_{0}^{4}, \\
& C_{1}=\frac{H_{0}^{2}\left(1+G_{x} H_{0}^{2}\right)}{G_{z}} .
\end{aligned}
$$

In the limit $H_{1} \rightarrow H_{0}, C_{0}$ represents pure gravity flow in the absence of capillary driving (i.e., when $\varepsilon=0$ or $h=H_{0}$ =const). There is a particular value of $G_{z}^{*}$ that halts the flow, for a given $\varepsilon$. By equating to zero the first two terms-not strictly correct for an asymptotic expansion-we find

$$
G_{z}^{*} \approx-\frac{\left(H_{0}-H_{1}\right)\left(1+G_{x} H_{0}^{2}\right)}{H_{0}^{2}} .
$$

Perhaps fortuitously, this result is correct to $\mathrm{O}(\varepsilon)$ as can be seen by comparing it to the exact expression, obtained by integrating Eq. (6) with $C=0$ (i.e., zero flow rate), 


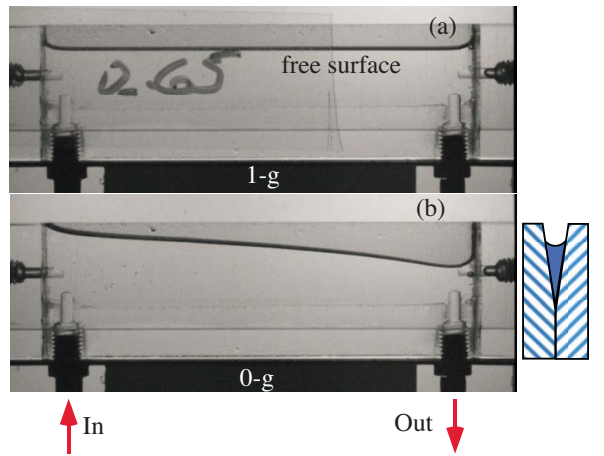

FIG. 3. (Color online) Images of steady flow experiments. (a) $1-g_{o}$. (b) Low- $g$. On the right, a schematic of the cross section.

$$
G_{z}^{*}=-\frac{\left(H_{0}-H_{1}\right)\left(1+G_{x} H_{0} H_{1}\right)}{H_{0} H_{1}} .
$$

Thus, for example, if $H_{0}>H_{1}\left(H_{0}<H_{1}\right)$, the capillary driving force is in the $+z(-z)$ direction and any $G_{z}<G_{z}^{*}<0$ $\left(G_{z}>G_{z}^{*}>0\right)$ reverses the flow.

\section{B. Comparison with steady flow experiments}

Before extending the analysis to unsteady flows we demonstrate the accuracy of the model by comparing the steady solutions with simple experiments. Direct comparisons are provided here for Eq. (24) $[z(h)]$ and Eq. (26) $(Q$ $\left.\equiv Q^{\prime} / W H^{2} \tan \alpha\right)$ with experiments performed in both terrestrial and reduced gravity environments. Only limited experimental verification of such flows has been provided in literature. $^{13}$

The experiment arrangement is a low-angle interior corner container oriented "horizontally" (i.e., $G_{x} \neq 0, G_{z}=0$ ), as pictured in Fig. 3. Liquid is pumped at a controlled flow rate into and out of a test cell $73 \mathrm{~mm}$ long and with half angle $\alpha=5^{\circ}$. The fluid is driven from left to right across the test cell by pressure gradients induced by surface tension and gravity. Fluids are polydimethyl siloxane (PDMS) (silicone oil) and their properties are listed in Table II.

For $5 \mathrm{cSt}$ PDMS at a flow rate $Q^{\prime}=0.0370 \mathrm{ml} / \mathrm{s}$, images are shown in Fig. 3 at steady state. The upper image is taken in a normal Earth gravity $\left(g_{o}\right)$, while the lower image is taken in the reduced gravity environment of an aircraft in free fall, ${ }^{14}$ where effectively $g<10^{-2} g_{o}$. Variations in the interface height $h(z)$ are hardly distinguishable in the terrestrial environment under these conditions. However, significant curvature is observed when gravity is reduced to the point $G_{x} \ll 1$. This limit is used to predict the now explicit interface profile $h(z)$ from Eq. (24) which is plotted in Fig. 4 alongside

TABLE II. Physical properties of PDMS fluids used in the experiments. $\nu$ is nominal kinematic viscosity.

\begin{tabular}{rcccc}
\hline \hline $\begin{array}{c}\nu \\
(\mathrm{cSt})\end{array}$ & $\begin{array}{c}\rho \\
\left(\mathrm{g} / \mathrm{cm}^{3}\right)\end{array}$ & $\begin{array}{c}\mu \\
(\mathrm{g} / \mathrm{cm} \mathrm{s})\end{array}$ & $\theta$ & $\begin{array}{c}\sigma \\
(\mathrm{dyn} / \mathrm{cm})\end{array}$ \\
\hline 5 & 0.913 & 4.56 & 0 & 19.7 \\
10 & 0.935 & 9.35 & 0 & 20.1 \\
\hline \hline
\end{tabular}

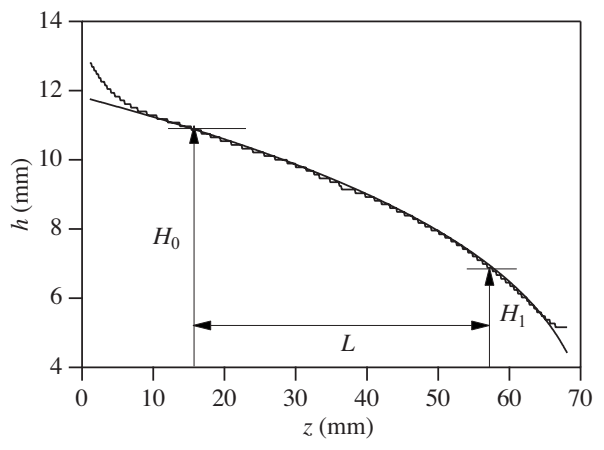

FIG. 4. $h^{\prime}\left(z^{\prime}\right)$ with $G_{x}=0$ from Eq. (24) plotted with pixelized experimental interface elevations. $Q_{\text {exp }}^{\prime}=0.037 \pm 0.005 \mathrm{ml} / \mathrm{s} ; 5 \mathrm{cSt}$ PDMS; channel half angle, $\alpha=5^{\circ}$. The portion of the measured interface used for fitting the theory is shown between $H_{0}$ and $H_{1}$.

the pixelized line of the empirically determined interface. Despite the excellent agreement $(<1 \%)$ for $h^{\prime}\left(z^{\prime}\right)$ over a large extent of the flow, the empirical inputs $H_{0}$ and $H_{1}$ in Eq. (26) for the prediction of $Q^{\prime}$ result in uncertainties of up to $10 \%$ due to their cubic dependence. The measured flow rates $Q_{\text {exp }}^{\prime}$ suffer in part from experimental uncertainty (for the low- $g$ apparatus $\leq 15 \%$ typical). Nonetheless, overpredictions from $1 \%$ to $25 \%$ are observed for flows satisfying the model assumptions. As an example, in the case of Fig. 4, an outlier value of $Q_{\exp }^{\prime}=0.037 \pm 0.005 \mathrm{ml} / \mathrm{s}$ is measured, while $Q^{\prime}$ determined from Eq. (26) is $0.046 \pm 0.004 \mathrm{ml} / \mathrm{s}$. Further terrestrial data for $G_{x} \lesssim 10$ is presented in Fig. 5 for various conditions and fluid properties. Using the same experiment apparatus, tests are performed for fixed fluid volume and varying flow rate $Q^{\prime}$ (left, $5 \mathrm{cSt} \mathrm{Si}$ oil), and for fixed flow rate and varying fluid volume (right, $10 \mathrm{cSt}$ Si oil). The volume condition is noted on the figures by a horizontal line indicating the mean liquid height (level) of the no-flow state. Equation (24) agrees well with the data. Flow rates predicted by Eq. (26) in general overpredict the experimental values by not more than $8 \%$. Calculated versus measured flow rates over a range of tests conducted are presented in Fig. 6. The systematic overprediction may be attributed to neglect of curvature in the $z$-direction and inertia, both of which retard the flow and increase with flow rate. The tests of the steady theory support the appropriateness of the model. In the rest of the paper we use the model to develop approximate unsteady flow solutions.

\section{UNSTEADY SOLUTIONS}

In this section we develop a perturbation theory for gravity effects on unsteady corner flows. Unlike the steady case, unsteady solutions cannot be obtained in closed form for arbitrary values of $G_{z}$ and $G_{x}$. Therefore we will pursue a perturbation analysis valid for small values of the gravity parameters $G_{z}$ (parallel to the flow) and $G_{x}$ (perpendicular to the flow). It is precisely this limit that is relevant in reduced gravity environments (i.e., spacecraft), where $g$ is finite but small. Assuming that both parameters are independent of one another, $h$ and $\mathcal{L}$ may be expanded asymptotically as 

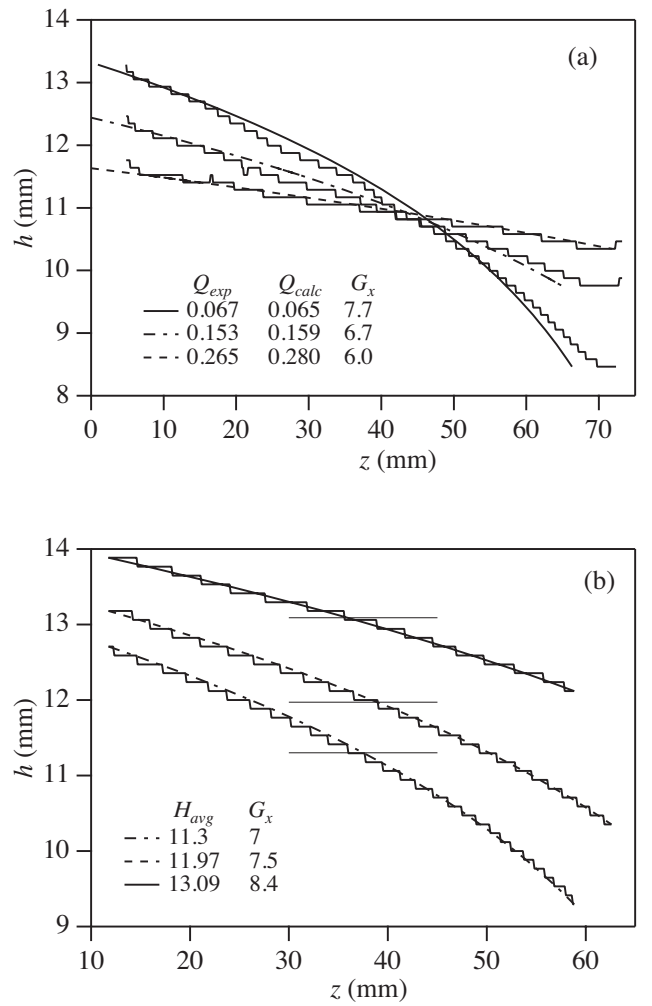

FIG. 5. Meniscus profile $h^{\prime}\left(z^{\prime}\right)$. Pixelized line: data; continuous line: Eq. (24). Corner half angle $\alpha=5^{\circ}$. All fits are based on the approximate $40 \mathrm{~mm}$ length region, $15 \mathrm{~mm}<z<55 \mathrm{~mm}$. (a) $h^{\prime}\left(z^{\prime}\right)$ for various flow rates $Q^{\prime}$ (noted in legend in $\mathrm{ml} / \mathrm{s}$ ) with volume fixed, $5 \mathrm{cSt}$ PDMS, $H_{\text {avg }}$ $=(11.09 \pm 0.07) \mathrm{mm}$. (b) $h^{\prime}\left(z^{\prime}\right)$ with constant flow rate $Q^{\prime}$ $=0.263 \pm 0.009 \mathrm{ml} / \mathrm{s}$, varying fluid volume as indicated by values of $H_{\text {avg }}$ listed in legend in mm. Fluid is $10 \mathrm{cSt}$ PDMS.

$$
h\left(z, \tau ; G_{x}, G_{z}\right) \sim h_{00}(z, \tau)+G_{x} h_{10}(z, \tau)+G_{z} h_{01}(z, \tau)+\cdots,
$$

$$
\mathcal{L}\left(\tau ; G_{x}, G_{z}\right) \sim \mathcal{L}_{00}(\tau)+G_{x} \mathcal{L}_{10}(\tau)+G_{z} \mathcal{L}_{01}(\tau)+\cdots
$$

as both $G_{x}, G_{z} \rightarrow 0$. In this notation, the first subscript refers to $G_{x}$ and the second one to $G_{z}$. Thus, 00 implies $G_{x}=G_{z}$ $=0,10$ denotes $G_{x} \neq 0$ and $G_{z}=0$, and 01 denotes $G_{x}=0$ and $G_{z} \neq 0$.

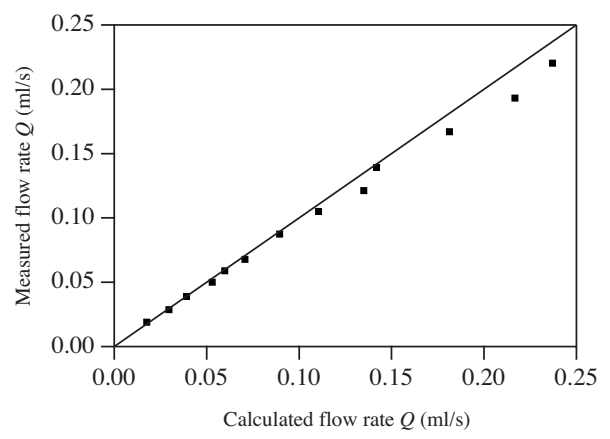

FIG. 6. Comparison of measured vs calculated flow rates [Eq. (26)] in normal Earth gravity $g_{o}$. Channel half angle, $\alpha=5^{\circ}$. Fluid, $5 \mathrm{cSt} \mathrm{Si}$ oil. Measured flow rate error is $< \pm 2 \%$. The black line has a $45^{\circ}$ slope.

\section{A. $\mathrm{O}(1)$ solution}

The equation for $h_{00}$ is the same one solved by W\&L and obtains by substituting Eq. (32) into Eq. (9) and equating the terms of $\mathrm{O}(1)$,

$$
h_{00_{\tau}}=2\left(h_{00_{z}}\right)^{2}+h_{00} h_{00_{z z}} .
$$

The tip height condition [Eq. (10)] at this order is

$$
h_{00}(z, \tau)=0 \quad \text { at } \quad z=\mathcal{L}_{00}(\tau) .
$$

If the injected volume at $z=0$ is prescribed, then the lowestorder version of Eq. (11) must be satisfied, viz.,

$$
V(\tau)=\int_{0}^{\mathcal{L}_{00}} h_{00}^{2} d z
$$

where we have assumed that the injected volume at $z=0$ may be a known function of $\tau$ but is independent of $G_{x}$ and $G_{z}$. If, on the other hand, the height at $\eta=0$ is prescribed as a function of time, then

$$
h_{00}(0, \tau)=\mathcal{H}(\tau)
$$

must be satisfied.

Equation (34) admits similarity solutions for a wide variety of volume time dependences [Eq. (36)] or boundary conditions at $z=0$ [Eq. (37)], many of which have been analyzed by W\&L and more completely in Ref. 15; here, we present only the essential results.

\section{Similarity}

If we define $F_{00}(\eta)$ by $h_{00}=\tau^{a_{00}} \lambda^{2} F_{00}(\eta)$, and $\eta=\lambda^{-1} \sqrt{-b_{00}} z \tau^{b_{00}}$, similarity is obtained if and only if $b_{00}=-\left(a_{00}+1\right) / 2$ and Eq. (34) becomes

$$
F_{00} F_{00}^{\prime \prime}+2\left(F_{00}^{\prime}\right)^{2}+\eta F_{00}^{\prime}-\frac{2 a_{00}}{1+a_{00}} F_{00}=0,
$$

where $\lambda$ is a factor that leaves this equation invariant and allows one to set $0 \leq \eta \leq 1$, and the prime denotes differentiation with respect to $\eta$. The value of $\lambda$ will be determined from the boundary conditions on $F_{00}$. The tip height condition [Eq. (35)] becomes

$$
F_{00}(1)=0 \text {, }
$$

and either using the tip condition Eq. (13) with Eqs. (32) and (33) or directly from local analysis of Eq. (38), we find the slope boundary condition

$$
F_{00}(1)=-\frac{1}{2} \text {. }
$$

Since $\eta=1$ at the tip, the definition of $\eta$ indicates that the tip location in physical space at this order is

$$
\mathcal{L}_{00}=\lambda \tau^{\left(a_{00}+1\right) / 2} \sqrt{\frac{2}{a_{00}+1}} .
$$

Thus, using conditions (39) and (40), Eq. (38) is integrated as an initial value problem from $\eta=1$ back to $\eta=0$ where the value of $F_{00}(0)$ is determined. 
TABLE III. Some physically relevant cases and special solutions of Eq. (34).

\begin{tabular}{cccccc}
\hline \hline$a_{00}$ & Boundary or volume condition & $F_{00}(\eta)$ & $F_{00}(0)$ & $F_{00}^{\prime}(0)$ & $\lambda$ \\
\hline 0 & $h_{00}(0)=\hat{\mathcal{H}}$ (constant height) & Numerical & 0.345 & -0.2051 & $\sqrt{\hat{\mathcal{H}} / F_{00}(0)}$ \\
1 & $h_{00}(0)=\hat{\mathcal{H}} \tau$ (constant rise) & $\frac{1-\eta}{2}$ & $\frac{1}{2}$ & $-\frac{1}{2}$ & $\sqrt{2 \hat{\mathcal{H}}}$ \\
$-\frac{1}{5}$ & $V=k$ (constant volume drop) & $\frac{1-\eta^{2}}{4}$ & $\frac{1}{4}$ & 0 & $(30 k)^{1 / 5}\left(\frac{2}{5}\right)^{1 / 10}$ \\
$\frac{1}{5}$ & $V=k \tau$ (constant source) & Numerical & 0.400 & -0.31165 & $\left(\frac{-k \sqrt{5}}{2 \sqrt{3} F_{00}^{2}(0) F_{00}^{\prime}(0)}\right)^{1 / 5}$ \\
$-\frac{1}{3}$ & $V=k \tau^{-1 / 3}$ (sink at $\left.z=0\right)$ & $\frac{3\left(\eta^{1 / 3}-\eta^{2}\right)}{10}$ & 0 & $\infty$ & $(500 k / 9 \sqrt{3})^{1 / 5}$ \\
\hline \hline
\end{tabular}

Knowing $F_{00}(\eta)$, the value of $\lambda$ is found from the similarity versions of either Eq. (36) and (37). If it is known that either $V_{00}(\tau)=k \tau^{m}$ [with $\left.m=\left(5 a_{00}+1\right) / 2\right]$ or $\mathcal{H}(\tau)=\hat{\mathcal{H}} \tau^{a_{00}}$, then $\lambda$ is found, respectively, from

$$
\begin{aligned}
\lambda^{5} \sqrt{\frac{2}{1+a_{00}}} \int_{0}^{1} F_{00}^{2} d \eta= & -\frac{4 \lambda^{5}}{5 a_{00}+1} \sqrt{\frac{a_{00}+1}{2}} \\
& \times F_{00}(0)^{2} F_{00}^{\prime}(0)=k
\end{aligned}
$$

or

$$
\hat{\mathcal{H}}=\lambda^{2} F_{00}(0) .
$$

By assigning values to $a_{00}$ we can create different physically relevant boundary or volume conditions for $h_{00}$. Table III summarizes the cases analyzed by W\&L; some of these cases were also analyzed by Cox and Verbist ${ }^{11}$ in the context of flow in foams in zero gravity. For the case $a_{00}=-1 / 3$, ours is a new analytical solution that had been until now calculated numerically. ${ }^{8}$ Details of the solution may be found in Appendix A.

\section{B. $O\left(G_{x}\right)$ solution, 10: Gravity perpendicular to channel}

At this order, Eq. (9) is

$$
\begin{aligned}
\frac{\partial h_{10}}{\partial \tau}= & h_{10} \frac{\partial^{2} h_{00}}{\partial z^{2}}+h_{00} \frac{\partial^{2} h_{10}}{\partial z^{2}}+4 \frac{\partial h_{00}}{\partial z} \frac{\partial h_{10}}{\partial z} \\
& +h_{00}^{2}\left[4\left(\frac{\partial h_{00}}{\partial z}\right)^{2}+h_{00} \frac{\partial^{2} h_{00}}{\partial z^{2}}\right] .
\end{aligned}
$$

The volume condition, the height condition at $z=0$, and the height condition at the tip may be written compactly as

$$
\begin{aligned}
& \int_{0}^{\mathcal{L}_{00}} h_{00} h_{i j} d z=0, \\
& h_{i j}(0)=0, \\
& h_{i j}\left(\mathcal{L}_{00}, \tau\right)+\left.\mathcal{L}_{i j} \frac{\partial h_{00}}{\partial z}\right|_{\left(\mathcal{L}_{00}, \tau\right)}=0
\end{aligned}
$$

for both orders $G_{x}(i j=10)$ and $G_{z}(i j=01)$. The height condition at the tip, Eq. (10), requires some care because $\mathcal{L}$ is part of the solution and a function of the small parameters of the problem. Thus, here we must expand both $h$ and $\mathcal{L}$. The latter demands that we expand $h\left(\mathcal{L}, \tau ; G_{x}, G_{z}\right)$ in a Taylor series about $\mathcal{L}_{00}$ in addition to applying the asymptotic expansion (32), to yield Eq. (47). This equation defines $\mathcal{L}_{i j}$, the correction to $\mathcal{L}_{00}$ at $\mathrm{O}\left(G_{x}\right)(i j=10)$ and at $\mathrm{O}\left(G_{z}\right)(i j=01)$.

\section{Similarity}

Equation (44) admits similarity solutions using the same independent variable, $\eta$, as in $\mathrm{O}(1)$. By defining

$$
h_{10}(z, \tau)=\tau^{3 a_{00}} \lambda^{6} F_{10}(\eta),
$$

$F_{10}(\eta)$ satisfies

$$
\begin{aligned}
& F_{10}^{\prime \prime} F_{00}+F_{10}^{\prime}\left(4 F_{00}^{\prime}+\eta\right)+F_{10}\left(F_{00}^{\prime \prime}-\frac{6 a_{00}}{a_{00}+1}\right) \\
& =-F_{00}^{2}\left(4 F_{00}^{\prime 2}+F_{00} F_{00}^{\prime \prime}\right) .
\end{aligned}
$$

It can be shown from Eq. (49) that at $\eta=1$,

$$
F_{10}^{\prime}+F_{10} \frac{17 a_{00}+1}{3\left(a_{00}+1\right)}=0 .
$$

The other condition for $F_{10}$ is either ${ }^{16}$

$$
\int_{0}^{1} F_{00} F_{10} d \eta=0
$$

or

$$
F_{10}(0)=0 \text {. }
$$

Once $F_{10}(\eta)$ is known, we may use Eq. (47) and definition (48) to get $\mathcal{L}_{10}$,

$$
\mathcal{L}_{10}=\frac{2^{3 / 2} \lambda^{5} \tau^{\left(5 a_{00}+1\right) / 2} F_{10}(1)}{\sqrt{a_{00}+1}} .
$$

Below we summarize the solutions of Eq. (49) for the cases examined by $\mathrm{W} \& \mathrm{~L}$ in the problem at $\mathrm{O}(1)$. All the cases that have exact solutions at $\mathrm{O}(1)$ have exact solutions at $\mathrm{O}\left(G_{x}\right)$ [and also at $\mathrm{O}\left(G_{z}\right)$ ]. Equation (49) satisfies boundary condition (50) and either the volume condition (51) or boundary condition (52), as stated below as "condition 2." 
(a) $a_{00}=1$ : Linearly increasing height at $\eta=0$.

- condition 2: Eq. (52),

- $F_{10}=\frac{1}{152}\left[1-3(\eta-1)+2(\eta-1)^{2}-\frac{1}{3}(\eta-1)^{3}\right]$,

- $F_{10}(1)=\frac{1}{152}$,

- $\mathcal{L}_{10}=\left[(2 \hat{\mathcal{H}})^{5 / 2} / 76\right] \tau^{3}$.

(b) $a_{00}=-\frac{1}{5}$ : drop of constant volume.

- condition 2: Eq. (51),

- $F_{10}=\frac{1}{320}\left[-1+7 \eta^{2}-\frac{17}{3} \eta^{4}+\frac{9}{5} \eta^{6}\right]$,

- $F_{10}(1)=\frac{1}{150}$,

- $\mathcal{L}_{10}=\frac{2}{5} k$.

(c) $a_{00}=-\frac{1}{3}$ : volume sink at $z=0$.

- condition 2: Eq. (51),

- $F_{10}=\left[9 /\left(25 \times 10^{3}\right)\right] \eta^{-2 / 3}\left(\eta^{1 / 3}-1\right)^{3}\left(27 \eta^{5 / 3}-2\right)(1$

$\left.+\eta^{1 / 3}+\eta^{2 / 3}+\eta+\eta^{4 / 3}\right)^{3}$

- $F_{10}(1)=0$,

- $\mathcal{L}_{10}=0$.

(d) $a_{00}=\frac{1}{5}$ : Constant flow rate at $z=0$.

- condition 2: Eq. (51),

- $F_{10}$ : numerical,

- $F_{10}(1) \approx 0.00439$,

- $\mathcal{L}_{10} \approx 0.0876\left(\frac{5}{3} k \tau\right)$.

(e) $a_{00}=0$ : constant height at $z=0$.

- condition 2: Eq. (52)

- $F_{10}$ : numerical

- $F_{10}(1) \approx 0.00886$,

- $\mathcal{L}_{10} \approx 0.13 \sqrt{2^{3} \hat{\mathcal{H}}^{5} \tau}$.

\section{C. $O\left(G_{z}\right)$ solution, 01 : Gravity parallel to the channel}

At this order, Eq. (9) is

$$
\frac{\partial h_{01}}{\partial \tau}=h_{01} \frac{\partial^{2} h_{00}}{\partial z^{2}}+h_{00} \frac{\partial^{2} h_{01}}{\partial z^{2}}+4 \frac{\partial h_{00}}{\partial z} \frac{\partial h_{01}}{\partial z}-4 h_{00}^{2} \frac{\partial h_{00}}{\partial z} \text {. }
$$

The volume condition, the height condition at $z=0$, and the height condition at the tip are shown in Eqs. (45)-(47), with $i j=01$.

\section{Similarity}

Equation (54) admits similarity solutions using the same independent variable, $\eta$, as in $\mathrm{O}(1)$. By defining

$$
h_{01}(z, \tau)=\tau^{\left(5 a_{00}+1\right) / 2} \lambda^{5} F_{01}(\eta),
$$

$F_{01}(\eta)$ satisfies

$$
\begin{aligned}
& F_{01}^{\prime \prime} F_{00}+F_{01}^{\prime}\left(4 F_{00}^{\prime}+\eta\right)+F_{01}\left(F_{00}^{\prime \prime}-\frac{5 a_{00}+1}{a_{00}+1}\right) \\
& =4 \sqrt{\frac{2}{a_{00}+1}} F_{00}^{2} F_{00}^{\prime} .
\end{aligned}
$$

It can be shown from Eq. (56) that at $\eta=1$,

$$
F_{01}^{\prime}+F_{01} \frac{14 a_{00}+4}{3\left(a_{00}+1\right)}=0 .
$$

In a completely analogous way to the $\mathrm{O}\left(G_{x}\right)$ problem, the second condition for $F_{01}$ is either ${ }^{17}$

$$
\int_{0}^{1} F_{00} F_{01} d \eta=0
$$

or

$$
F_{01}(0)=0 \text {. }
$$

Once $F_{01}(\eta)$ is known, we may use Eq. (47) and definition (55) to get $\mathcal{L}_{01}$,

$$
\mathcal{L}_{01}=\sqrt{\frac{8}{a_{00}+1}} \lambda^{4} \tau^{2 a_{00}+1} F_{01}(1) .
$$

Below we summarize the solutions of Eq. (56) for the cases examined by $W \& L$ in the problem at $O(1)$. All the cases that have exact solutions at $\mathrm{O}(1)$ have exact solutions at $\mathrm{O}\left(G_{z}\right)$. Equation (56) satisfies boundary condition (57) and either the volume condition (58) or boundary condition (59), as stated below as condition 2 .

(a) $a_{00}=1$ : Linearly increasing height at $\eta=0$.

- Condition 2: Eq. (59),

- $F_{01}=(\eta / 76)\left(11-16 \eta+6 \eta^{2}\right)$,

- $F_{01}(1)=\frac{1}{76}$,

- $\mathcal{L}_{01}=2 \hat{\mathcal{H}}^{2} \tau^{3} / 19$.

(b) $a_{00}=-\frac{1}{5}$ : drop of constant volume.

- Condition 2: $\int_{-1}^{1} F_{00} F_{01} d \eta=0$. Note that the effect of gravity on the constant volume drop is antisymmetric about $z=0$. This is opposite to the case when gravity points perpendicular to the channel, where the effect is symmetric about $z=0$. Thus, here the volume condition must be taken between -1 and 1 because gravity hastens one end of the drop and slows down the other. Thus, $F_{01}(\eta)$ (from which the correction $h_{01}$ is obtained) and the tip position correction at $\mathrm{O}\left(G_{z}\right)$ must both be antisymmetric about $\eta=0$,

- $F_{01}=\sqrt{10} / 840 \eta\left[19-16 \eta^{2}+5 \eta^{4}\right]$,

- $F_{01}(1)=-F_{01}(-1)=\sqrt{10 / 105}$,

- $\mathcal{L}_{01}^{ \pm}= \pm \frac{2}{21}\left(\frac{2}{5}\right)^{2 / 5} 30^{4 / 5} \tau^{3 / 5} k^{4 / 5}$, where the + and - refer to the drop tips at $z>0$ and $z<0$, respectively. The tip at $z>0$ moves with gravity; the tip at $z<0$ moves against gravity.

(c) $a_{00}=-1 / 3$ : Volume sink at $z=0, \quad V=k \tau^{m}, m=\left(5 a_{00}\right.$ $+1) / 2=-1 / 3$.

- Condition 2: Eq. (58),

- $F_{01}=\eta^{1 / 3} g(\eta)$ where 


$$
\begin{aligned}
g(x) \equiv & C+\frac{1}{523600}\left\{2244 x^{14 / 3}-\frac{19754 x^{3}}{3}\right. \\
& +6174 x^{4 / 3}+300 \sqrt{2}(\sqrt{5-\sqrt{5}} \\
& +\sqrt{5+\sqrt{5}}) \tan ^{-1}\left(\frac{4 x^{1 / 3}-\sqrt{5}+1}{\sqrt{2(5+\sqrt{5})}}\right) \\
& +300 \sqrt{2}(\sqrt{5-\sqrt{5}} \\
& -\sqrt{5+\sqrt{5}}) \tan ^{-1}\left(\frac{4 x^{1 / 3}+\sqrt{5}+1}{\sqrt{2(5-\sqrt{5})}}\right) \\
& -300 \sqrt{5} \ln \frac{x^{2 / 3}+\frac{1}{2}(1-\sqrt{5}) x^{1 / 3}+1}{x^{2 / 3}+\frac{1}{2}(1+\sqrt{5}) x^{1 / 3}+1} \\
& \left.+\frac{375 x^{2 / 3}\left(x^{2 / 3}-1\right)}{x^{5 / 3}-1}-\frac{625}{x}\right\}
\end{aligned}
$$

and $C \approx 1.89 \times 10^{-3}$.

- $F_{01}(1) \approx 0.00442352$,

- $\mathcal{L}_{01} \approx 1.7 k^{4 / 5} \tau^{1 / 3}$.

(d) $a_{00}=\frac{1}{5}$ : Constant flow rate at $z=0$.

- Condition 2: Eq. (58),

- $F_{01}$ : numerical.

- $F_{01}(1) \approx 3.59 \times 10^{-2}$,

- $\mathcal{L}_{01} \approx 0.394\left(\frac{5}{3}\right)^{9 / 10} 2^{1 / 5} k^{4 / 5} \tau^{7 / 5}$.

(e) $\quad a_{00}=0$ : Constant height at $z=0$.

- Condition 2: Eq. (59),

- $F_{01}$ : numerical,

- $F_{01}(1) \approx 2.17 \times 10^{-2}$,

- $\mathcal{L}_{01} \approx 0.182 \times 2^{3 / 2} \hat{\mathcal{H}}^{2} \tau$.

\section{Special case: Exponential height at $z=0$}

When $h\left(0, \tau ; G_{x}, G_{z}\right)=\mathcal{H} \exp \left(a_{00} \tau\right)$, W\&L (Ref. 8) generate a similarity equation at $\mathrm{O}(00)$ by writing $h_{00}(z, \tau)$ $=\exp \left(a_{00} \tau\right) \lambda^{2} F_{00}(\eta)$, where $\eta \equiv \lambda^{-1} \sqrt{-b_{00}} z \exp \left(b_{00} \tau\right)$. Similarity demands that $b_{00}=-a_{00} / 2$. The resulting equation and relation between $b_{00}$ and $a_{00}$ are the same that were obtained, respectively, from Eq. (38) and $b_{00}=-\left(a_{00}+1\right) / 2$ as the leading order in the asymptotic limit $a_{00} \rightarrow \infty$.

At $\mathrm{O}\left(G_{x}\right)$ and $\mathrm{O}\left(G_{z}\right)$ similarity is obtained with $h_{10}=\exp \left(3 a_{00}\right) \lambda^{6} F_{10}(\eta)$ and $h_{01}=\exp \left(\frac{5}{2} a_{00}\right) \lambda^{5} F_{01}(\eta)$, respectively.

$F_{i j}(\eta)$ obeys either Eq. (49) or Eq. (56); and $\mathcal{L}_{i j}$ follows expressions analogous to Eqs. (53) and (60), all derived as leading orders in the asymptotic limit of $a_{00} \rightarrow \infty$,

$$
\begin{aligned}
& \mathcal{L}_{10}=2^{3 / 2}\left(a_{00}\right)^{-1 / 2} \lambda^{5} \exp \left(\frac{5 a_{00}}{2} \tau\right) F_{10}(1), \\
& \mathcal{L}_{01}=16\left(a_{00}\right)^{-1} \lambda^{4} \exp \left(2 a_{00} \tau\right) F_{01}(1)
\end{aligned}
$$

The value of $\lambda$ is found from the boundary condition, $h(0, \tau)=\mathcal{H} \exp \left(a_{00} \tau\right)$,

$$
\lambda=\sqrt{\frac{\mathcal{H}}{F_{00}(0)}} .
$$

From integration of Eq. (38) with $F_{00}(1)=0, F_{00}^{\prime}=-1 / 2$, we find $F_{00}(0) \approx 0.637$. Since the boundary condition at $z=0$ specifies the height, we find after perturbation that $F_{i j}(0)$ $=0$ for both $i j=10$ and $i j=01$ [see Eqs. (52) and (59)]. Thus,

$$
\begin{aligned}
\mathcal{L}_{10} & =5.25 \times 10^{-2}\left(a_{00}\right)^{-1 / 2} \mathcal{H}^{5 / 2} \exp \left(\frac{5 a_{00}}{2} \tau\right), \\
\mathcal{L}_{01} & =0.12\left(a_{00}\right)^{-1} \mathcal{H}^{2} \exp \left(2 a_{00} \tau\right) .
\end{aligned}
$$

\section{DISCUSSION}

\section{A. Steady solution}

The steady solution with gravity can only be found implicitly as $z=\mathbb{Z}\left(h, H_{0}, H_{1}, L ; G_{x}, G_{z}\right)$. We have generated solutions that contain the foam drainage equation (FDE) solutions as special cases that correspond to a channel of infinite length and $G_{x}=0$. Our analysis covers all possible flow direction and liquid slope combinations.

The flow rate depends in a complex way on the inlet and outlet heights $H_{0}$ and $H_{1}$, respectively. This dependence may be expressed by the difference $H_{0}-H_{1}$ only when $H_{0} \approx H_{1}$. When $G_{z}=0$, the flow rate depends on $H_{0}^{3}-H_{1}^{3}$ and $H_{0}^{5}-H_{1}^{5}$, see Eq. (24).

Experiments with steady flow support the dependence of $z$ on $G_{x}$ found in the model for a wide range $0 \leq G_{x} \lesssim 10$. The dependence on $G_{z}$ has been tested extensively in the context of the FDE. ${ }^{10}$ Although the solutions relevant for foam drainage are different from those we address here, tests of the model are interchangeable.

\section{B. Unsteady solutions}

We have developed solutions for cases where the boundary condition at $z=0$ gives rise to a simple expression in the similarity domain. In addition to these cases, a wide selection of height or volume conditions may be computed using the appropriate $a_{00}$. Then, using the procedure outlined here, $F_{i j}(\eta)$ is obtained and $\mathcal{L}_{10}$ and $\mathcal{L}_{01}$ follow from Eqs. (53) and (60), respectively. This problem allows for the higher orders $\mathrm{O}\left(G_{x}\right)$ and $\mathrm{O}\left(G_{z}\right)$ to be analyzed using similarity by preserving the independent variable $\eta$ that appears at $\mathrm{O}(1)$, and with an exponent of $\tau$ that is uniquely determined by $a_{00}$.

At $\mathrm{O}\left(G_{x}\right)$, the gravity pointing down onto the meniscus makes the meniscus less curved in the channel cross section, an effect analogous to what happens to a sessile drop sitting on top of a horizontal surface in a gravitational field. One might think that this reduction in the meniscus curvature would slow down the corner capillary flow since in the extreme case of "infinite" gravity the meniscus is flat and the capillary driving force disappears. However, another consequence of vertical gravity is the development of a hydrostatic pressure gradient down the channel proportional to $G_{x} d h / d x$; and the net effect of the two is to hasten the flow. Examination of the solution to $\mathrm{O}\left(G_{x}\right)$, Eq. (53), shows that for $F_{10}(1) \neq 0, \mathcal{L}_{10}$ is independent of $\tau$ when $a_{00}=-1 / 5$; and $\mathcal{L}_{10}$ 
decreases (or increases) with $\tau$ as $a_{00}$ is less than (or larger than) $-1 / 5$. In the special case $a_{00}=-1 / 3, F_{10}(1)=0$ [see (c) in Sec. IV B 1) and the correction to the tip position due to perpendicular gravity is identically zero, even though $a_{00}=-1 / 3$ is in the "increasing with $\tau$ " domain based on the previous discussion.

At $\mathrm{O}\left(G_{z}\right)$ when gravity is parallel to the channel axis, similarity solutions can also be found for all values of $a_{00}$. The gravity effect on the constant volume drop is now antisymmetric about $z=0$, with the forward (relative to gravity) tip being accelerated and the rear tip (which moves against gravity) being slowed down by gravity.

As discussed in Sec. II, the value of the geometrical factor $F_{i}$ depends on $G_{x}$ through the effect that $G_{x}$ has on the cross sectional area shape. Thus, strictly speaking, we should have expanded $F_{i}$ in an asymptotic series in $G_{x}$. However, when $G_{x}=0, F_{i}$ is most sensitive to the corner angle $\alpha$; a good approximation is

$$
F_{i} \approx \frac{1}{6}\left(1-\frac{\sin 2 \alpha}{5}\right) \pm 2 \% \text { standard deviation, }
$$

which does not even contain the contact angle and shows a weak $(<20 \%)$ variation with $\alpha$. Since the effect of $G_{x}$ is to flatten the cross sectional meniscus, analogous to the contact angle $\theta \rightarrow \pi / 2-\alpha$, we assume that the effect of $G_{x}$ is of similar order to that of $\theta$. Therefore Eq. (67) is considered valid even when $G_{x} \neq 0$ and, thus, $F_{i}$ is taken to be independent of $G_{x}$ in the analysis. Using Eq. (67), we find the bound $1 / 8 \leq F_{i} \leq 1 / 6$ introduced by W\&L (Ref. 8) for all values of $\alpha$ and $\theta$.

The relevance of solutions to the FDE to the problem of capillary flow in corners needs further discussion in addition to the arguments offered in the Introduction. The case presented in Ref. 10 of a traveling wave front of liquid imbibing an initially dry foam may look like an analog of a liquid fillet advancing into a dry corner channel. However there are fundamental differences between the two. First, the traveling wave-by its very nature-has an upstream condition of constant liquid area at upstream "infinity." This implies that, upstream, the flow cannot be driven by capillarity and must be entirely gravity driven. Second, similarity in the case of gravity along the channel without perturbation in $G_{z}$ only obtains for $a_{00}=b_{00}=-1 / 3$. This case corresponds to a liquid volume sink at $z=0$ not to a constant liquid area upstream. The similarity equation that is obtained in this case does not admit solutions that asymptote to a constant at upstream infinity. Thus, we conclude that the class of similarity solutions presented in Ref. 15, from which our equations can be deduced, is fundamentally different from the class of nonsimilarity solutions presented in Ref. 10.

In summary, we have analyzed the capillary corner flow in a sharp corner under weak gravity. We have examined the important practical limits of gravity perpendicular and parallel to the channel corner. In the asymptotic limit $G_{x} \ll 1, G_{z}$ $\ll 1$, the two effects are linearly independent and their combined effect is obtained by linear superposition. The perturbed problems for the gravity-driven corrections to the zero-gravity case may be reduced to similarity using the same independent similarity variable as in the zero-gravity problem. Not surprisingly, the effect of gravity is to hasten the capillary flow when it points down into the channel and when it points along the flow direction. The main results of this work are expressions useful for system design and analysis of the gravity corrections to the liquid tip position in the channel as a function of time presented in Secs. IV B 1 and IV $\mathrm{C} 1$. Even though the analysis necessitated the introduction of $a d$ hoc scales $H$ and $L$, the dimensional results presented in Appendix B are naturally free of these parameters and involve only fluid properties and information from the volume or flux/height condition at $z=0$. Finally, a new analytic solution to an equation describing nonlinear unsteady heat conduction has been derived in the context of the case $a_{00}=-1 / 3$. The details may be found in Appendix A.

\section{APPENDIX A: SOLUTION TO $F_{00}$ FOR $a_{00}=-1 / 3$}

The equation to be solved is

$$
F_{00} F_{00}^{\prime \prime}+2\left(F_{00}^{\prime}\right)^{2}+\eta F_{00}^{\prime}+F_{00}=0,
$$

subject to

$$
F_{00}(1)=0 \quad \text { and } \quad \lim _{\eta \rightarrow 0} F_{00}^{\prime} F_{00}^{2}=\text { bounded } .
$$

The second of the above conditions arises from the fact that the flux, $Q=h_{z} h^{2}$, at $z=0$ must be bounded.

This equation is the similarity version of a special case of a nonlinear heat equation,

$$
\frac{\partial T}{\partial t}=A \frac{\partial}{\partial z}\left(T^{n} \frac{\partial T}{\partial z}\right)
$$

which is obtained for $n=1 .{ }^{18}$ Local analysis of Eq. (A1) near $\eta=0$ gives $F_{00} \sim \eta^{1 / 3}$ as $\eta \rightarrow 0$. We now define an auxiliary function $f(\eta) \equiv \eta^{-1 / 3} F_{00}(\eta)$, with bounded $f^{\prime}(0)$, which obeys

$$
3 \eta f f^{\prime \prime}+6 f^{\prime}(\eta f)^{\prime}+3 \eta^{5 / 3} f^{\prime}+4 \eta^{2 / 3} f=0 .
$$

Rearranging,

$$
f\left(3 \eta f^{\prime \prime}+6 f^{\prime}+4 \eta^{2 / 3}\right)+f^{\prime}\left(6 \eta f^{\prime}+3 \eta^{5 / 3}\right)=0 .
$$

This can be an exact differential only if

$$
\frac{d}{d \eta}\left(6 \eta f^{\prime}+3 \eta^{5 / 3}\right)=3 \eta f^{\prime \prime}+6 f^{\prime}+4 \eta^{2 / 3},
$$

or, upon simplification,

$$
f^{\prime}+\frac{1}{2} \eta^{2 / 3}=0 .
$$

When this condition is met, we may rewrite Eq. (A5) as

$$
\frac{d}{d \eta}\left[f\left(6 \eta f^{\prime}+3 \eta^{5 / 3}\right)\right]=0 .
$$

Integrating once,

$$
f\left(6 \eta f^{\prime}+3 \eta^{5 / 3}\right)=\mathrm{C} .
$$

Since $\mathrm{C}$ is a constant, and $\mathrm{C}=0$ when $\eta=0$, it follows that $\mathrm{C} \equiv 0$. Thus, either $f \equiv 0$ (trivial solution, physically not admissible) or 


$$
2 f^{\prime}+\eta^{2 / 3}=0
$$

This is the same as the condition needed to make Eq. (A5) an exact differential. Thus, the maneuver is self-consistent and the solution to Eq. (A10) is also a solution of Eq. (A5). Integrating Eq. (A10) and demanding that $F_{00}(1)=f(1)=0$ yields

$$
F_{00}=\frac{3}{10}\left(\eta^{1 / 3}-\eta^{2}\right)
$$

This solution meets the two conditions stated in Eq. (A2).

When $a_{00}<-1 / 3$, the solution $F_{00}$ to Eq. (38) does not remain strictly positive in $0 \leq \eta \leq 1$. Thus, Eq. (A11) provides an exact solution to the lower bound of strictly positive similarity solutions in $0 \leq \eta \leq 1$.

\section{APPENDIX B: DIMENSIONAL FORMS FOR MENISCUS HEIGHT AND LENGTH}

In this section we give expressions for the meniscus height as a function of axial position and time, and the liquid length as a function of time. Expressing each dimensionless variable, $h, z, t\left(\equiv 2 \tau / F_{i}\right), \mathcal{L}$, as the corresponding dimensional counterpart divided by the appropriate scale indicated in Sec. II, and after tedious but straightforward algebra, it is possible to show that the horizontal and vertical length scales, $L, H$, drop out of the problem. This should happen since these flows do not have a physical length scale.

Below we summarize the dimensional results for each of the cases analyzed in the paper, identified by the value of $a_{00}$. In the expressions that follow, $F_{00}(\eta), F_{10}(\eta), F_{01}(\eta)$ must be used with the corresponding expressions given in Secs. IV B 1 and IV C 1. For convenience, we drop the primes with the understanding that all variables in this section are dimensional. Coefficients shown as real numbers with decimal points are the result of numerical calculations.

It is convenient to define the parameters,

$$
l_{c} \equiv\left(\frac{\sigma}{f \rho g}\right)^{1 / 2}, \quad G \equiv \frac{\sigma}{\mu} \frac{F_{i} \sin ^{2} \alpha}{f}
$$

(a) $a_{00}=1$ : height at $z=0$ linear with time, $h(0, t)=k t$,

$$
\begin{aligned}
h= & 2 k t F_{00}(\eta)+8 l_{c}^{-2} \cos \gamma k^{3} t^{3} F_{10}(\eta) \\
& +4 G^{1 / 2} l_{c}^{-2} \sin \gamma k^{5 / 2} t^{3} F_{01}(\eta), \\
\mathcal{L}= & (k G)^{1 / 2} t+\frac{1}{19} G^{1 / 2} l_{c}^{-2} \cos \gamma k^{5 / 2} t^{3} \\
& +\frac{1}{19} G^{1 / 2} l_{c}^{-2} \sin \gamma k^{2} t^{3} .
\end{aligned}
$$

Here,

$$
\eta=\frac{z}{t(k G)^{1 / 2}}
$$

(b) $a_{00}=-1 / 5$ : constant volume drop, $V=k\left(2 H^{2} L F_{A}\right)$,

$$
\begin{aligned}
h= & \left(\frac{15 V}{F_{A}}\right)^{2 / 5}\left(\frac{4}{5 G t}\right)^{1 / 5} F_{00}(\eta) \\
& +\left(\frac{15 V}{F_{A}}\right)^{6 / 5}\left(\frac{4}{5 G t}\right)^{3 / 5} l_{c}^{-2} \cos \gamma F_{10}(\eta) \\
& +4\left(\frac{15 V}{F_{A}}\right) l_{c}^{-2} \sin \gamma F_{01}(\eta) \\
\mathcal{L}= & \left(\frac{15 V}{F_{A}}\right)^{1 / 5}\left(\frac{5}{4} G t\right)^{2 / 5} \\
& +\frac{1}{5} l_{c}^{-2} \frac{V}{F_{A}} \cos \gamma \pm \frac{2}{5} l_{c}^{-2}\left(\frac{5}{4} G t\right)^{3 / 5}\left(\frac{15 V}{F_{A}}\right)^{4 / 5} \sin \gamma
\end{aligned}
$$

Here,

$$
\eta=z\left(\frac{4}{5 G t}\right)^{2 / 5}\left(\frac{F_{A}}{15 V}\right)^{1 / 5} .
$$

In the expression for $\mathcal{L}$, the gravity along the channel pointing in the $+z$ direction has an antisymmetric effect, lengthening the drop in $z>0$ and shortening it in $z<0$, compared to the no-gravity case. The plus and minus refer to the positive and negative ends of the drop, respectively.

(c) $a_{00}=-1 / 3$ : sink at $z=0, V=k t^{-1 / 3}$,

$$
\begin{aligned}
h= & \left(\frac{500 k}{9 \sqrt{3} F_{A}}\right)^{2 / 5}\left(\frac{2}{G}\right)^{1 / 5} t^{-1 / 3} F_{00}(\eta) \\
& +l_{c}^{-2}\left(\frac{2}{G}\right)^{3 / 5}\left(\frac{500 k}{9 \sqrt{3} F_{A}}\right)^{6 / 5} t^{-1} \cos \gamma F_{10}(\eta) \\
& +l_{c}^{-2}\left(\frac{500 k}{9 \sqrt{3} F_{A}}\right) t^{-1 / 3} \sin \gamma F_{01}(\eta), \\
\mathcal{L}= & \left(\frac{500 k}{F_{A}}\right)^{1 / 5}\left(\frac{G}{2}\right)^{2 / 5} t^{1 / 3} \\
& +0.106 l_{c}^{-2}\left(\frac{500 k}{9 \sqrt{3} F_{A}}\right)^{4 / 5}\left(\frac{G}{2}\right)^{3 / 5} \sin \gamma t^{1 / 3} .
\end{aligned}
$$

Here,

$\eta=\frac{z}{t^{1 / 3}}\left(\frac{F_{A}}{500 k}\right)^{1 / 5}\left(\frac{2}{G}\right)^{2 / 5}$.

In this curious case, there is no contribution to $\mathcal{L}$ from gravity perpendicular to the channel, identified by the term proportional to $\cos \gamma$, and associated with the order $\mathrm{O}\left(G_{x}\right)$ [see $(\mathrm{c})$ in Sec. IV B 1$]$.

(d) $a_{00}=1 / 5$, constant flow rate $Q$ at $z=0, V=Q t$,

$$
\begin{aligned}
h= & 3.31\left(\frac{10 t}{3 G}\right)^{1 / 5}\left(\frac{Q}{2 F_{A}}\right)^{2 / 5} F_{00}(\eta) \\
& +36.34 l_{c}^{-2}\left(\frac{10 t}{3 G}\right)^{3 / 5}\left(\frac{Q}{2 F_{A}}\right)^{6 / 5} \cos \gamma F_{10}(\eta) \\
& +19.97 l_{c}^{-2}\left(\frac{5}{3}\right)^{1 / 2}\left(\frac{Q t}{2 F_{A}}\right) \sin \gamma F_{01}(\eta),
\end{aligned}
$$




$$
\begin{aligned}
\mathcal{L}= & 1.82\left(\frac{Q}{2 F_{A}}\right)^{1 / 5}\left(\frac{G}{2}\right)^{2 / 5}\left(\frac{5 t}{3}\right)^{3 / 5}+8.76 \\
& \times 10^{-2} l_{c}^{-2}\left(\frac{Q}{F_{A}}\right) \cos \gamma\left(\frac{5 t}{3}\right)+7.42 \\
& \times 10^{-2} 2^{1 / 5}\left(\frac{5}{3}\right)^{9 / 10} l_{c}^{-2}\left(\frac{Q}{F_{A}}\right)^{4 / 5}\left(\frac{G}{2}\right)^{3 / 5} \sin \gamma t^{7 / 5} .
\end{aligned}
$$

Here,

$$
\eta=\frac{1}{1.82}\left(\frac{2 F_{A}}{Q}\right)^{1 / 5}\left(\frac{2}{G}\right)^{2 / 5} z\left(\frac{3}{5 t}\right)^{3 / 5} .
$$

(e) $a_{00}=0$, constant height $\mathcal{H}$ at $z=0$,

$$
\begin{aligned}
h= & 2.9 \mathcal{H} F_{00}(\eta)+24.31 \mathcal{H}^{3} l_{c}^{-2} \cos \gamma F_{10}(\eta) \\
& +14.3 \mathcal{H}^{5 / 2} l_{c}^{-2}\left(\frac{G t}{2}\right)^{1 / 2} \sin \gamma F_{01}(\eta), \\
\mathcal{L}= & 1.702(\mathcal{H} G t)^{1 / 2}+0.13 \times 2 \mathcal{H}^{5 / 2} l_{c}^{-2}(G t)^{1 / 2} \cos \gamma \\
& +0.182 \times 2^{1 / 2} \mathcal{H}^{2} l_{c}^{-2} G t \sin \gamma .
\end{aligned}
$$

Here,

$$
\eta=\frac{z}{1.702}\left(\frac{1}{\mathcal{H} G t}\right)^{1 / 2} \text {. }
$$

${ }^{1} \mathrm{P}$. Concus and R. Finn, "On the behavior of a capillary surface in a wedge," Proc. Natl. Acad. Sci. 63, 292 (1969).

${ }^{2}$ P. S. Ayyaswamy, I. Catton, and D. K. Edwards, "Capillary flow in triangular grooves," ASME Trans. J. Appl. Mech. 41, 332 (1974).

${ }^{3}$ T. C. Ransohoff and C. J. Radke, "Laminar flow of a wetting liquid along the corners of a predominantly gas-occupied noncircular pore," J. Colloid Interface Sci. 121, 392 (1988).

${ }^{4}$ M. Dong and I. Chatzis, "The imbibition and flow of a wetting liquid along the corners of a square capillary," J. Colloid Interface Sci. 172, 278 (1995).

${ }^{5}$ L. A. Romero and F. G. Yost, "Flow in an open channel capillary," J. Fluid Mech. 322, 109 (1996).

${ }^{6}$ A. R. Kovscek and C. J. Radke, "Gas bubble snap-off under pressuredriven flow in constricted noncircular capillaries," Colloids Surf., A 117, 55 (1996).

${ }^{7}$ M. M. Weislogel, "Capillary flow in an interior corner," Ph.D. thesis, Northwestern University, 1996.

${ }^{8}$ M. M. Weislogel and S. Lichter, "Capillary flow in an interior corner," J. Fluid Mech. 373, 349 (1998).

${ }^{9} \mathrm{~J}$. Bico and D. Quéré, "Rise of liquids and bubbles in angular capillary tubes," J. Colloid Interface Sci. 247, 162 (2002).

${ }^{10}$ G. Verbist, D. Weaire, and A. M. Kraynik, "The foam drainage equation," J. Phys.: Condens. Matter 8, 3715 (1996).

${ }^{11}$ S. J. Cox and G. Verbist, "Liquid flow in foams under microgravity," Microgravity Sci. Technol. 14, 45 (2003).

${ }^{12}$ M. M. Weislogel, "Some analytical tools for fluids management in space: Isothermal capillary flows along interior corners," Adv. Space Res. 32, 163 (2003).

${ }^{13}$ J. Klatte, D. Haake, M. M. Weislogel, and M. E. Dreyer, "A fast numerical procedure for steady capillary flow in open capillary channels," Acta Mech. 201, 269 (2008).

${ }^{14}$ R. C. McKnight, E. S. Neumann, and J. P. Withrow, "Reduced gravity experimental capabilities of NASA Lewis' DC-9 Aircraft," AIAA 34th Aerospace Sciences Meeting, Reno, NV, January 1996, AIAA Paper No. 96-0921.

${ }^{15}$ A. D. Polyanin and V. F. Zaitsev, Handbook of Nonlinear Partial Differential Equations (Chapman \& Hall/CRC, Boca Raton, 2004).

${ }^{16}$ Instead of evaluating Eq. (51), it is sometimes simpler to evaluate the left-hand side of Eq. (16) at $\mathrm{O}\left(G_{x}\right)$ and express it in terms of the similarity variables: $-\left.F_{00}\left(F_{00}^{3} F_{00}^{\prime}+2 F_{10} F_{00}^{\prime}+F_{10} F_{00}^{\prime}\right)\right|_{\eta=0}=\left[\left(9 a_{00}+1 / a_{00}\right.\right.$ $+1)] \int_{0}^{1} F_{00} F_{10} d \eta$.

${ }^{17}$ From Eq. (16) it can be shown that Eq. (58) implies $F_{00}^{4}$ $+\sqrt{a_{00}+1 / 2}\left(2 F_{01} F_{00}^{\prime}+F_{00} F_{01}^{\prime}\right)=0$ at $\eta=0$.

${ }^{18}$ F. J. Mayer, J. F. McGrath, and J. W. Steele, "A class of similarity solutions for the nonlinear thermal conduction problem," J. Phys. A 16, 3393 (1983). 
Physics of Fluids is copyrighted by the American Institute of Physics (AIP). Redistribution of journal material is subject to the AIP online journal license and/or AIP copyright. For more information, see http://ojps.aip.org/phf/phfor.jsp 